\title{
A molecular perspective of water at metal interfaces
}

\author{
Javier Carrasco ${ }^{1}$, Andrew Hodgson ${ }^{2}$, Angelos Michaelides ${ }^{3}$ \\ ${ }^{1}$ Instituto de Catálisis y Petroleoquímica, CSIC, \\ Marie Curie 2, E-28049, Madrid, Spain \\ ${ }^{2}$ Surface Science Research Centre and Department of Chemistry, \\ University of Liverpool, Oxford Street, Liverpool, L69 3BX, UK \\ ${ }^{3}$ Thomas Young Centre, London Centre for Nanotechnology and Department of Chemistry, \\ University College London, London WC1E 6BT, UK
}

\begin{abstract}
Water-solid interfaces are ubiquitous and of the utmost importance to industry, technology and many aspects of daily life. Despite countless studies from different areas of science, detailed molecular-level understanding of water-solid interfaces comes mainly from well-defined studies on flat metal surfaces. These studies have recently shown that a remarkably rich variety of structures form at the interface between water and seemingly simple flat metal surfaces. Here we discuss some of the most exciting examples of recent work in this area and the underlying physical insight and general concepts that emerge about how water binds to surfaces. A perspective on the outstanding problems, challenges, and open questions in the field is also provided.

Copyright (c) 2012, Rights Managed by Nature Publishing Group
\end{abstract}




\section{INTRODUCTION}

Water-solid interfaces are important to an incredibly broad range of everyday phenomena and scientific and technological processes. This includes areas such as corrosion, electrochemistry, nanoparticle self-assembly, environmental chemistry, lubricants, and heterogeneous catalysis, to name just a few. Indeed the ubiquitous presence of water on surfaces under ambient conditions means that water-solid interfaces are relevant to many areas of the physical sciences. In addition, contemporary issues such as climate change and shortages in both water and energy mean that there is a pressing need to better understand the structure and dynamics of water-solid interfaces. This, along with fundamental questions about hydrogen ( $\mathrm{H})$ bonding and $\mathrm{H}$ atom transfer ${ }^{1,2}$, has resulted in a flurry of interest in recent years and the chemistry and physics of interfacial water is now one of the most exciting and thriving fields in materials chemistry and physics.

The first step towards understanding water-solid interfaces is the challenging task of knowing where the atoms and molecules are located. This is challenging because of the extremely small number of atoms at the interface (relative to the bulk) and the difficulty of analyzing the details of atomic-level geometries and electronic structure for such a small sample $^{3}$. Typically this requires what is generally referred to as a surface science style approach, i.e., extremely clean, atomically smooth substrates are interrogated under ultrahigh vacuum (UHV) conditions to determine the arrangement of water and how it bonds to the surface. Of course we do not live under UHV conditions, but if we want to obtain precise atomic-level understanding and rigorously "solve" water-surface structures, UHV provides the armoury of tools we need. A disadvantage of this approach is that water is only stable at cryogenic temperatures (up to about $200 \mathrm{~K}$ ) in UHV, so that the structures which form are frozen, without any of the dynamic disorder of a liquid. A second experimental limitation is that often only the first contact layer is examined, with few experiments able to probe thicker water layers in any detail. A consequence of this is that understanding how the interface structure responds to multilayer adsorption, or to melting, requires us to develop accurate, predictive theoretical models for the solid water interface based on the few systems studied in detail. Thus, although there is broad interest in water-solid interfaces from many disparate areas of science, the fundamental atomic-level insight into water-solid interfaces is currently limited to a small number of surface science style studies, some of which we 
discuss in this article.

Probably the biggest conceptual idea to emerge from well-defined studies of water at interfaces is that of the bilayer adsorption model. The bilayer model is a concept that has been used to discuss interfacial water in almost all environments and is often the first concept that newcomers to the field grasp. Perhaps as a consequence, the bilayer model has gained a currency that is entirely out of line with the experimental evidence in its favour, often being the last concept to be abandoned only in the face of overwhelming evidence! The term bilayer is used both in its strict sense - a buckled hexagonal overlayer resembling the basal plane of ice - and more loosely to discuss structuring of water films at interfaces where two peaks in the density profile are observed. The bilayer model was originally developed based on experiments on water at metal surfaces and this article will explore some of the groundbreaking new work published in this area recently which calls this model for water adsorption into question. Indeed, here we will consider if a water bilayer in the strict sense of the word has ever been observed, or should really be expected.

In the following we will explore what the bilayer model is, how it emerged, and how recent experiments and calculations reveal a much more interesting variety and richness of structure for water at interfaces. The focus will be on water-metal interfaces, the system where the first structural models for water at interfaces emerged and where some of the most detailed and exciting recent experiments and simulations have been performed. Key recent developments include the observation of wetting layer structures built exclusively from pentagons, or from combinations of heptagons and pentagons, the observation of wetting layer structures rich in defects in the $\mathrm{H}$ bonded network, the recognition of the importance of strain in the $\mathrm{H}$ bonded network, and the realisation that maximising the number of $\mathrm{H}$ bonds does not necessarily lead to the most stable overlayer structures. The challenges and issues that remain before a fully predictive understanding of the structure and dynamics of watersolid interfaces can be achieved will also be discussed. This will be a short review aimed mainly at newcomers to the field, using a few selected examples to illustrate the concepts and exciting developments alluded to above. For comprehensive overviews of the literature up to 2009 the interested reader is referred to refs. [4,5] and for other complementary mini-reviews on some specific aspects of water at surfaces to refs. $\left[\underline{6}^{-} \underline{\underline{8}}\right]$. 


\section{A BRIEF HISTORY OF WATER ON METALS: THE RISE OF THE BILAYER}

As we have said, obtaining atomic-level understanding of surface structure is very challenging. For water-solid interfaces this generally involves preparing clean atomically flat surfaces upon which water is deposited under UHV conditions. This frozen in structure typically a single layer — can then be interrogated with, for example, techniques such as low energy electron diffraction (LEED). In the 1980s Doering and Madey performed such a study ${ }^{\underline{9}}$, examining in meticulous detail water adsorbed on the close-packed (0001) surface of $\mathrm{Ru}$. This surface has an hexagonal arrangement of metal atoms with the distance between neighboring $\mathrm{Ru}$ atoms within $3 \%$ of the nearest neighbour water-water distance in ice I. Upon observing a $\sqrt{3} \times \sqrt{3}-R 30^{\circ}$ LEED pattern the bilayer model was introduced for extended 2D overlayer adsorption of water on metals. The bilayer model proposed by Doering and Madey ${ }^{9}$ consists of an epitaxial 2D arrangement of water molecules forming an hexagonal network, which resembles the (001) basal plane of ice I (see Fig. 1). It is referred to as a bilayer simply because the hexagonal rings within the (001) basal plane of ice are puckered with molecules at two distinct heights. At a metal surface it was suggested that the water molecules within the lower part of the bilayer interact relatively strongly with the surface, whereas the water molecules in the upper part of the bilayer (about $1 \AA$ higher) are $\mathrm{H}$ bonded to the bottom layer and do not interact appreciably with the surface. In a conventional bilayer the molecules in the top half of the bilayer have $\mathrm{OH}$ bonds which point away from the surface, often referred to as dangling $\mathrm{OH}$ groups or the H-up bilayer model. An alternative arrangement in which the $\mathrm{OH}$ groups are directed at the surface ("H-down") has also been suggested ${ }^{5} \underline{10}$. In either case it's interesting to note that these are so called proton ordered structures when built within a two molecule $\sqrt{3}$ unit cell and as such are bilayers of ice XI, the proton ordered form of ice I.

Following Doering and Madey, $\sqrt{3}$ LEED patterns observed on other metal surfaces $\frac{5}{5}$ were interpreted as evidence of the generality of the bilayer model. In addition an apparent correlation between lattice mismatch and binding energy — obtained by monitoring desorption temperatures of water from various metals — was taken as further evidence for an epitaxial bilayer in which the intermolecular water-water distances adapted to the lattice constant of the underlying substrate ${ }^{11}$. The bilayer model was also adapted to explain the $(2 \times 2)$ diffraction patterns observed on f.c.c. (110) surfaces $^{5}$. Essentially the bilayer model 
(c)

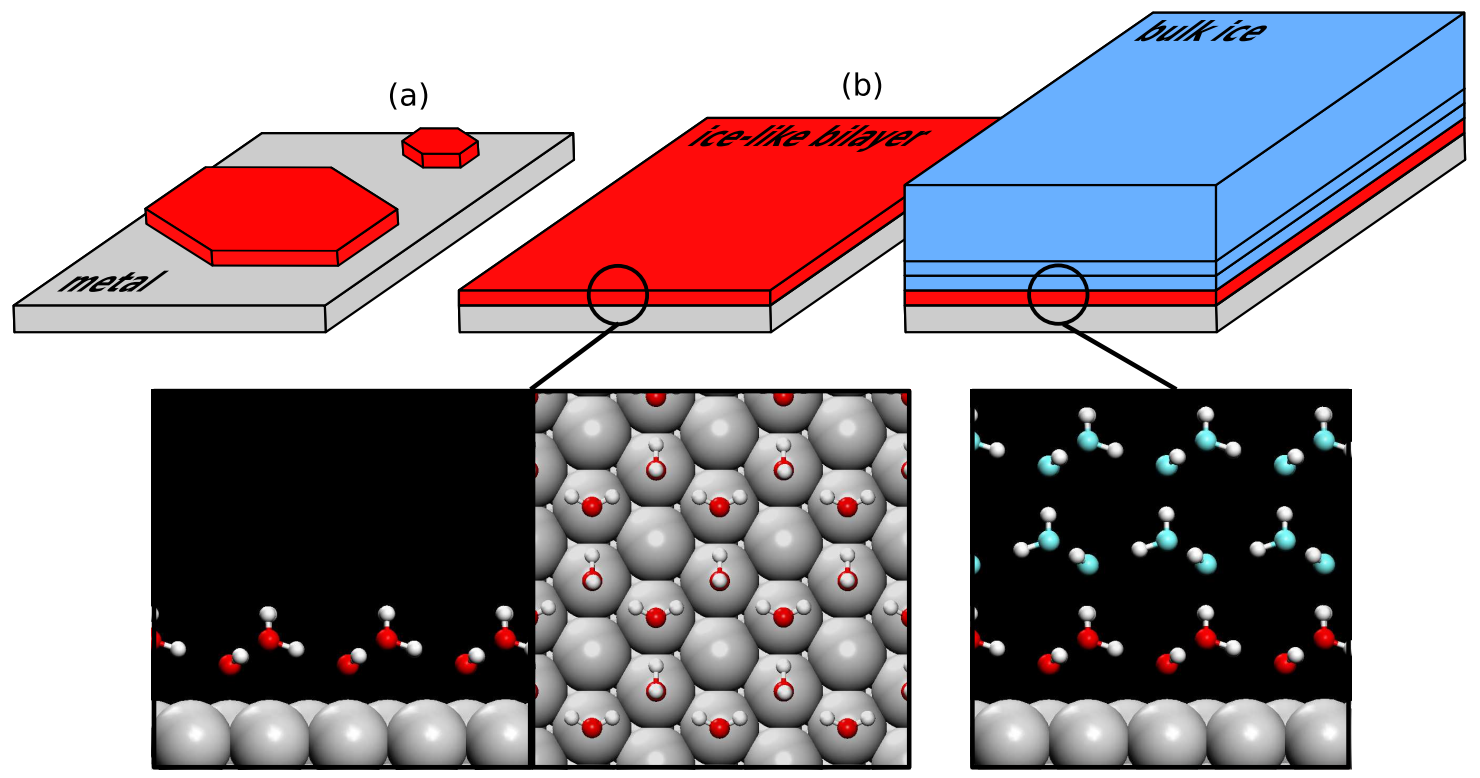

FIG. 1: Schematic (upper frames) and molecular models of the traditional model for water/ice adsorption on a solid surface. (a) Water is assumed to form extended 2D islands of the ice bilayer structure that (b) forms a wetting layer in registry with the close packed surface. Ice multilayers then grow on top of the first layer, forming a strained epitaxial arrangement (c).

became the standard model for water adsorption and was subsequently used to interpret surface structures on all sorts of inorganic substrates. It is still widely used.

\section{THE FIRST SIGNS OF A PROBLEM...}

In the 1990s Held and Menzel reported a more sophisticated LEED measurement that led to the first structural characterization of a water overlayer on any surface ${ }^{12}$. Using a newly developed low current LEED apparatus they performed what's known as a LEED-IV study. By comparing LEED-IV spectra with simulated spectra obtained from trial structural models, a 3D structural model of the interface can be arrived at describing the location of the heavy atoms at the interface. (Because of the light mass of hydrogen their positions are not readily determined in $\mathrm{LEED}^{13}$, another reason why in general solving structures for water is difficult). Held and Menzel found that the best fit to their data was obtained when the oxygen atoms were almost co-planar. This non-buckled "monolayer" is the antithesis of a bilayer wherein the $\mathrm{O}$ atoms are buckled by almost $1 \AA$ to gain the tetrahedral coordination 
found in ice I. Thus the very first structural characterisation of water at an atomically flat interface conducted almost 20 years ago was actually inconsistent with the bilayer model.

One of the first people to think about this apparent contradiction was Feibelman $\frac{14}{}$ who in 2002 reported density functional theory (DFT) calculations for water on Ru. He suggested that the small buckling was a sign that some of the water molecules in the overlayer had dissociated yielding a so called partially dissociated overlayer, similar to an overlayer reported earlier for water on $\operatorname{Pt}(111)^{15}, \underline{16}$. Feibelman's calculations indicated that partially dissociated structures containing both $\mathrm{OH}$ and $\mathrm{H}_{2} \mathrm{O}$ are significantly more stable than any conventional intact bilayer picture and suggested that what Held and Menzel had observed was a partially dissociated overlayer. This suggestion prompted a flurry of interest and water on Ru quickly became the hottest system for investigation in the 2000s. The reader interested in the details of this controversial and at times heated discussion is referred to Hodgson's review $\stackrel{5}{\underline{5}}$. For the present discussion it is sufficient to say that, somewhat ironically, the $\sqrt{3}$ diffraction pattern responsible for the birth of the bilayer model does not actually result from a conventional bilayer $\underline{\underline{17}}$.

\section{THE STM REVOLUTION: THE FALL OF THE BILAYER}

With the development of new techniques - most notably scanning tunnelling microscopy (STM) - it became possible to simply "see" at a local level what structures water forms when it sticks to metal surfaces. Such experiments, when interpreted together with DFT (and sometimes also with spectroscopic or diffraction measurements), have transformed our understanding of water-solid interfaces. A selection of recent low temperature STM results for water on metals is shown in Fig. 2, One of the most interesting points to note from

all these experiments is the tremendous diversity of structures observed. Although the experiments were all performed at low temperatures on atomically flat metal surfaces, on each surface a different structure is observed. On some surfaces (generally at lower coverages) isolated water clusters are observed. On others, 1D or quasi-1D chains are observed, on others $2 \mathrm{D}$ overlayers with mixtures of pentagons and heptagons, and on others 3D pyramids and towers. In no case is a uniform 2-D ice bilayer detected. We now discuss some of the systems shown in Fig. 2 and the concepts and physical understanding that have emerged. 


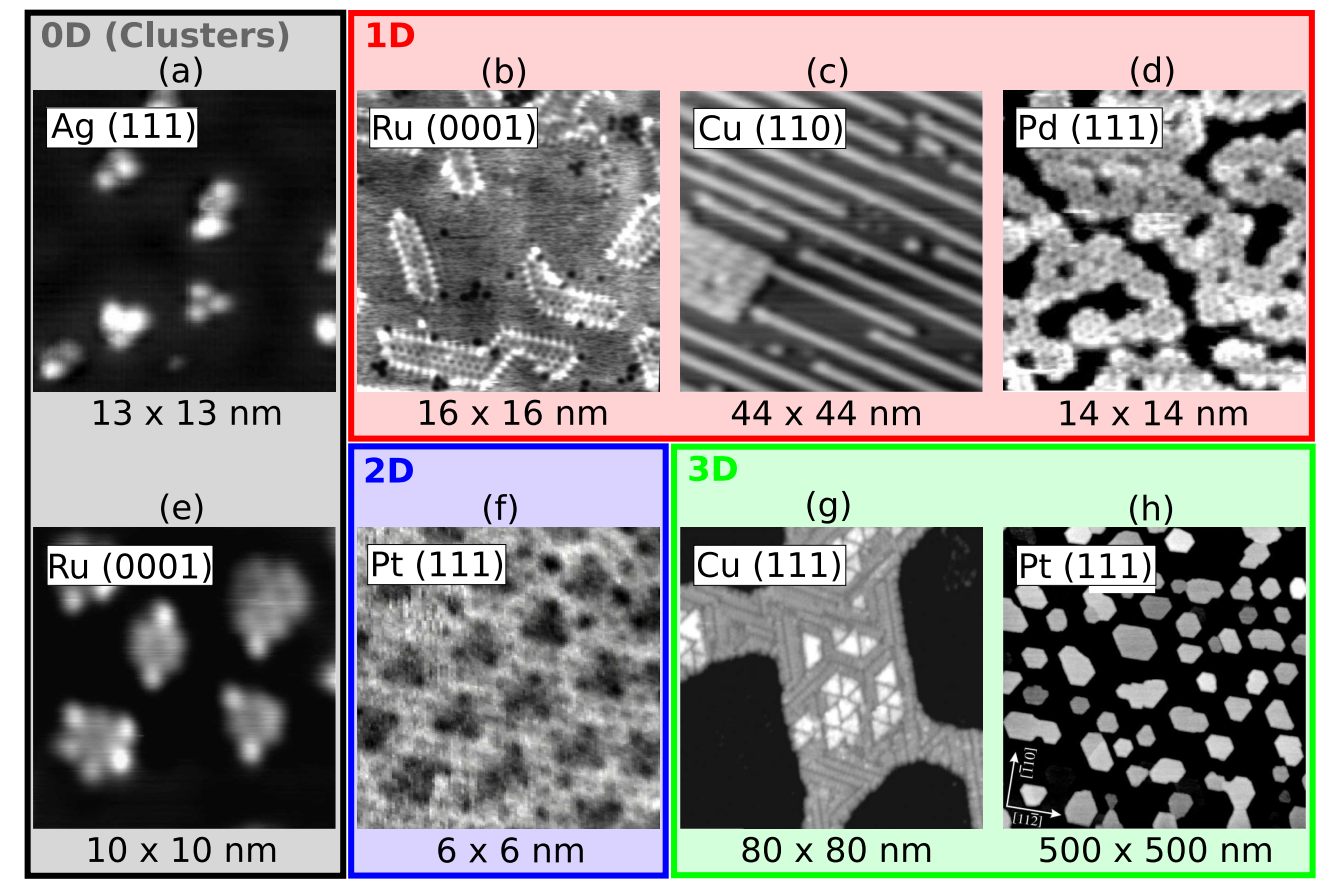

FIG. 2: Experimental STM images of water clusters and overlayers on metals. A selection of structures with different dimensionality are shown: (a,e) clusters of about 6 to 20 water molecules on $\operatorname{Ag}(111)$ and $\operatorname{Ru}(0001)$; (b,c,d) strips, long chains, and rosette meshes, (f) 2D network, and (g,h) crystalline 3D pillars and pyramids. Adapted from Gawronski et al. 18 (a), Tatarkhanov et al..$^{19}(\mathrm{~b})$, Yamada et $a l^{20}$ (c), Cerda et $a l^{21}$ (d), Salmeron et al. ${ }^{22}$ (e), Nie et al. ${ }^{23}$ (f), Mehlhorn et al. ${ }^{24}(\mathrm{~g})$, and Thürmer and Bartelt 25 (h).

\section{A. Water clusters: "Zero-dimensional" structures}

Individual water molecules are extremely difficult to image on metal surfaces due to their high mobility and preference to form $\mathrm{H}$ bonded clusters. Water monomers can be identified at very low coverage and temperatures (below $20 \mathrm{~K})^{2,26,27}$, sitting in the atop sites (i.e., the sites directly above individual metal atoms of the substrate). The atop sites were also predicted to be the most stable adsorption sites with density functional theory (DFT) on both close-packed and more open metal surfaces ${ }^{28}-30$. At this adsorption site, DFT further predicts that the dipole moment of the water molecule is aligned almost parallel to the surface (Fig. 3(c)). This binding mode favours interaction of the $1 b_{1}$ molecular orbital of water with the surface 28,31 .

Even at these low temperatures, however, water diffuses rapidly across metal surfaces and 



FIG. 3: Experimental STM images and geometrical models predicted by theory for a water monomer and water clusters on various metal surfaces. The experimental images correspond to a water monomer on $\mathrm{Cu}(110)$ (a), a water dimer on $\mathrm{Pt}(111)(\mathrm{d})$, and a water hexamer on $\mathrm{Cu}(111)(\mathrm{g})$. Top and side views of the corresponding theoretical structures are shown in the central and right hand columns. It has been suggested that the flower-like shape in the experimental STM image of the water dimer (d) is a consequence of rapid helicopter-like rotation of the water

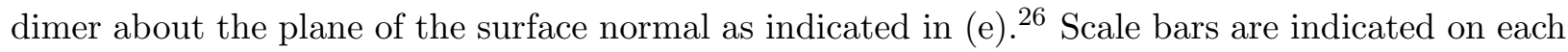
of the images where useful, either reporting distances in Angstrom ((d), (e), (g), (h)) or in terms of the underlying lattice constant $\left(\mathrm{a}_{0}\right.$, panels $(\mathrm{a})$ and $\left.(\mathrm{b})\right)$. Adapted from Okuyama et al. ${ }^{32}$ (a), Motobayashi et al. $\underline{26}(\mathrm{~d})$, and Michaelides and Morgenstern $\underline{1}$ (g).

readily forms $\mathrm{H}$ bonded clusters. The simplest cluster, the water dimer, has been observed on a number of metal surfaces $\underline{26}, 33$ and DFT studies suggest that it has an asymmetric structure with the two water molecules at different heights above the surface (Fig. 3(f)). The water molecule donating the $\mathrm{H}$ bond is more tightly bound and closer to the surface (generally by about $0.5 \AA$ ) than the water molecule accepting the $\mathrm{H}$ bond. It has been suggested that this asymmetric structure facilitates facile $\mathrm{H}$ bond rearrangement, rotation, and diffusion of 
water dimers $\underline{8}, 26,29,33$.

Adsorbed water trimers and tetramers have all also been observed ${ }^{32}, 34$, with the tetramer being the first cyclic cluster that is more stable than a simple linear chain on $\mathrm{Cu}(110)$. However, the cluster which has received the most attention is the cyclic water hexamer. Interest in hexamers stems partly from the fact that they are seen as the building blocks of ice and, in the past few years, hexamers have been successfully resolved with STM (often in conjunction with complementary DFT studies) on $\mathrm{Pd}, \mathrm{Cu}$, and $\mathrm{Ag}$ surfaces $1,18,35-38$. Although in the gas phase water hexamers possess a number of almost iso-energetic isomeric forms (notably the "cage", "book", "prism", and "cyclic"' isomers 39 ), when observed on metal surfaces only cyclic water hexamers have been detected to date. In these hexamers each water molecule is located near the favoured atop adsorption site and accepts and donates just a single $\mathrm{H}$ bond. Adsorbed cyclic hexamers, therefore, have a different $\mathrm{H}$ bonding topology to a hexamer extracted from a bilayer of ice (c.f. Figs. 1 and 3(h)).

Depending on the substrate, the hexamers are either planar (with all molecules at the same height) or buckled (with molecules at two distinct heights, as shown in Fig. 3(i)). Generally planar hexamers are favoured on reactive surfaces to which water molecules bond relatively strongly (e.g. $\mathrm{Ru})^{40}$, whereas on the noble metals $(\mathrm{Cu} \text { and } \mathrm{Ag})^{\underline{1}}$ the buckled hexamer is favoured. This difference points to the subtle balance between water-water and water-metal interactions in directing the structure of these adsorption systems. Indeed, as we will see, this balance is the single most important issue in determining the structure of water clusters and overlayers on metals. In the DFT calculations of water hexamers on $\mathrm{Cu}$ and $\mathrm{Ag}$ the balance between water-water and water-metal interactions was examined in detail and it was shown that there was a competition between the ability of water molecules to simultaneously bond to a substrate and to accept $\mathrm{H}$ bonds. This comes about because the same molecular orbital involved in bonding to the surface $\left(1 b_{1}\right)$ is also implicated in the acceptance of $\mathrm{H}$ bonds. This competition was in turn used to rationalize previous structure predictions for other water clusters adsorbed on various surfaces, including the asymmetric buckled structure of the water dimers and higher clusters ${ }^{1}$. Loosely speaking, we can think of planar hexamers as optimizing the interaction between the water and the metal on reactive surfaces, whereas buckled structures optimize the $\mathrm{H}$ bonding within the cluster on noble metals. As we will see shortly, the formation of planar networks on reactive surfaces places constraints on how large a water cluster can grow before the structure is forced to buckle 
out of plane, reducing the bonding to the surface.

\section{B. One-dimensional structures}

We move our attention now to structures with periodicity in one dimension. To this end we focus on recent studies of $c a .1 \mathrm{~nm}$ wide ice chains that nucleate on $\mathrm{Cu}(110) \underline{20,41,42}$. A particularly striking example of one of the chains — which form spontaneously when water is adsorbed on $\mathrm{Cu}(110)$ at submonolayer coverages — is shown in Fig. 4. Early suggestions for the structure of the chains were based on a bilayer-like arrangement of water molecules or other hexagonal structures ${ }^{20}$. However, it was subsequently shown, through a combination of STM, infrared spectroscopy and DFT, that the chains are not built from hexagons, but instead are built from a face-sharing arrangement of water pentagons $\frac{42}{2}$. Although there is a precedent for pentagon-based ice structures in other environments $\underline{43}, \underline{44}$, this was the first time that such a structural unit had been seen at a surface. The nucleation of pentagons revealed an unanticipated structural adaptability of water-ice films at surfaces, which goes well beyond the simple bilayer model.

The pentagon structure is favoured over others (e.g. the traditional hexamer building block) because it maximises the water-metal bonding while maintaining a strong $\mathrm{H}$ bonding network. Specifically, in the pentagon chains two thirds of the water molecules bond at atop adsorption sites of the $\mathrm{Cu}$ ridges, a higher proportion of molecules at the preferred adsorption sites than found in other structures. In addition, the pentagon structure allows for the strongest $\mathrm{H}$ bonded network with minimal strain within the overlayer. Indeed, fitting a commensurate network of hexagons across the 110 rows of the $\mathrm{Cu}$ substrate as chains would require a c.a. $1 \AA$ compression of each hexagon compared to the ideal hexagonal geometry of ice. This introduces strain in the overlayer, which thus destabilizes structures based on hexagons. Put simply, the slighter smaller pentagons make a better fit to the $\mathrm{Cu}$ substrate than do hexagons.

With the above in mind DFT was also used to predict the structure and relative stability of $1 \mathrm{D}$ chains of hexagons and pentagons on the (110) surfaces of $\mathrm{Ni}, \mathrm{Pd}$ and $\mathrm{Ag}$. Along with $\mathrm{Cu}$, this consists of a set of four (110) substrates with a separation between the close-packed

110 ridges that ranges from about $3.5 \AA$ to $4.2 \AA$. As shown in Fig. 4(c) an interesting correlation between the relative stability of hexagon versus pentagon chains was observed. 

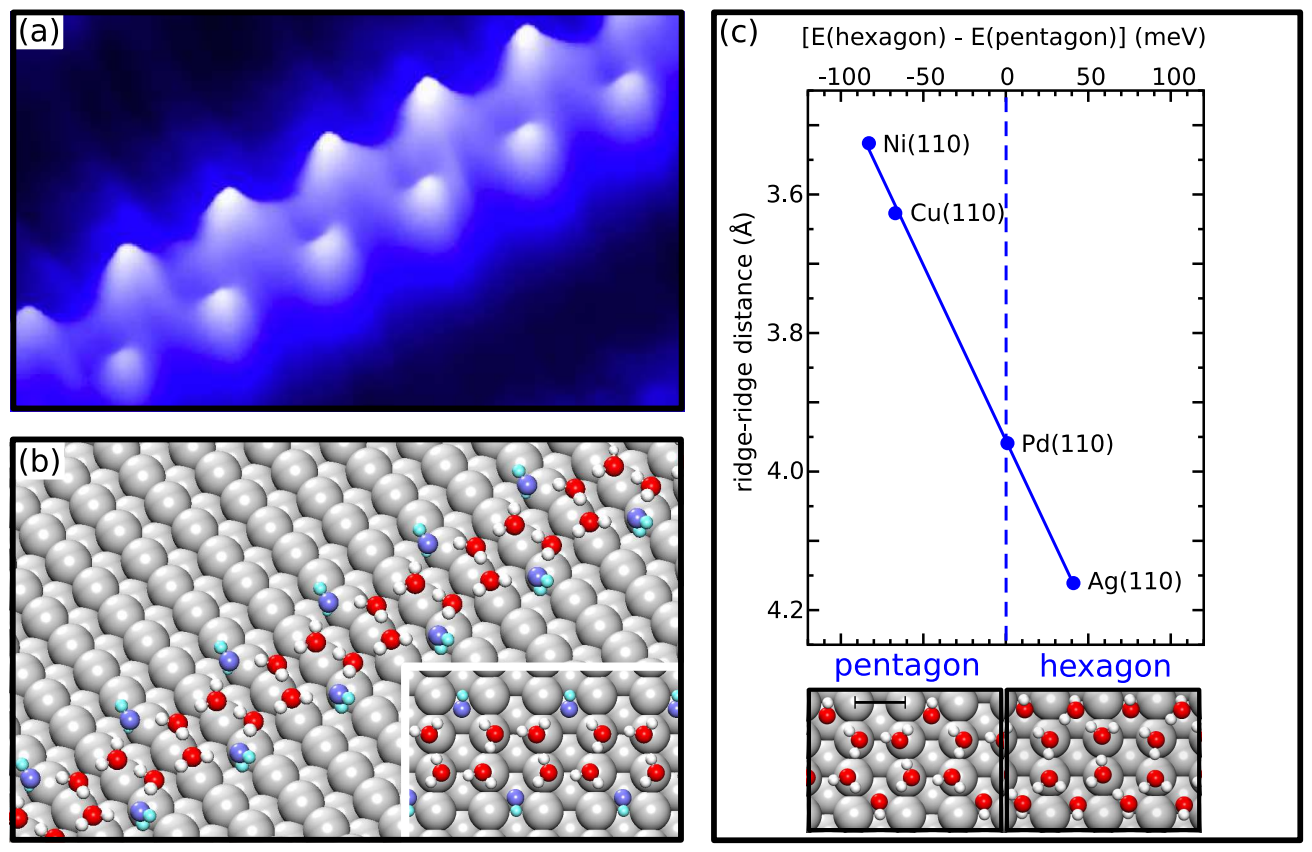

FIG. 4: STM and DFT results for water on $\mathbf{C u ( 1 1 0 ) . ~ ( a ) ~ S T M ~ i m a g e ~ o f ~ t h e ~ c a . ~} 1 \mathrm{~nm}$ wide ice chains that nucleate on $\mathrm{Cu}(110)$. (b) Structural model derived for the chains, which comprises a zig-zag arrangement of water pentagons. It is the blue water molecules in (b) that give rise to the brightest features in (a). (c) Illustration of how the relative stability (in $\mathrm{meV} / \mathrm{H}_{2} \mathrm{O}$ ) of hexagon and pentagon based 1D chains depends on the lattice constant of the underlying substrate (expressed in terms of the distance between the close-packed 110 ridges. This shows that according to DFT pentagons are favoured on substrates with a relatively small lattice constant ( $\mathrm{Ni}$ and $\mathrm{Cu}$ ), whereas hexagons are favoured on the substrate with the largest lattice constant, Ag. Figure based on results reported in ref. $\stackrel{42}{.}$.

Pentagons are favoured on substrates with small lattice constants; that is, in addition to $\mathrm{Cu}(110), \mathrm{Ni}(110)$ is identified as a substrate on which pentagons may nucleate. $\operatorname{Pd}(110)$ is a borderline case, whereas on the substrate with the largest lattice constant $\operatorname{Ag}(110)$, there is a small preference for hexagons. This trend holds for the same water structures in the gas phase (that is, without the presence of the substrate) ${ }^{42}$, which provides further support that $\mathrm{H}$ bonding is key to the relative stability of hexagons versus pentagons.

The water networks that form when about 0.5 ML of water is dosed on the close-packed (111) of $\mathrm{Pd}$ at low temperature are an intriguing example of a structure that is intermediate between $1 \mathrm{D}$ and 2D, as shown in Fig. 2(d). The water/Pd system has already been 
reviewed ${ }^{5,7,8,45}$ and so we point out just the key features of this system here. Essentially this structure is mainly comprised of long chains of hexagonal units, whose width never exceeds a few hexagonal cells, as shown in Fig. 2(d). When one uses DFT to work out the structure of the chains it turns out that the overlayer is again not based on the puckered hexagonal structure of ice I. Instead, it involves an arrangement of water molecules wherein most of the molecules are at their preferred atop adsorption sites in their preferred orientation almost parallel to the surface. Having water molecules flat against the surface places too many O-H bonds in the plane parallel to the surface and so a structure dominated by flat lying water molecules is only possible in the submonolayer regime with, in this case, a highly defective, metastable $\mathrm{H}$ bonded network. Completing the network forms a complex, partially ordered 2D structure made up of flat and H-down water $\underline{46}$.

\section{Two-dimensional structures}

The 2D water monolayer that covers $\mathrm{Pt}(111)$ at low temperature ${ }^{47}$ is the perfect example of an extended overlayer which clearly deviates from the ice-like bilayer picture. After a long standing effort, in 2010 two independent studies were able to obtain high resolution STM images of this water layer ${ }^{23} \underline{48}$ (see Fig. 5(a)). The current interpretation of this data, essentially supported by DFT calculations, seems to indicate that the wetting layer on $\mathrm{Pt}(111)$ is a mixture of pentagonal, hexagonal, and heptagonal rings of water molecules $\underline{23}, \underline{49}$ (Fig. 5(b)). Similar to the isolated hexamers and 1D chains in previous sections, the balance between water-metal and water-water interactions drives the $\mathrm{H}$ bonding structure adopted. The unit cell contains ca. $26 \mathrm{H}_{2} \mathrm{O}$ molecules, where just 6 are lying flat on atop Pt sites; the rest are bonded well above the surface and are tilted out of the surface plane. This layer has a substantial fraction of dangling $\mathrm{H}$ bonds, mostly pointing down towards the surface, and preserves a complete $\mathrm{H}$ bonding network. Overall this configuration appears to be favoured by allowing a small fraction of the water molecules to bind flat, close to the metal substrate, maximizing their water-metal interaction while still completing a perfect $\mathrm{H}$ bonded network of water molecules.

The wetting of $\mathrm{Cu}(110)$ offers another recent example where the ice-like bilayer model does not hold. Above $140 \mathrm{~K}$ experiments indicate the formation of a partially dissociated water layer, that is ordered yet not fully stoichiometric (with ca. 2:1 $\mathrm{H}_{2} \mathrm{O}-\mathrm{OH}$ ratio) ${ }^{5}$. 

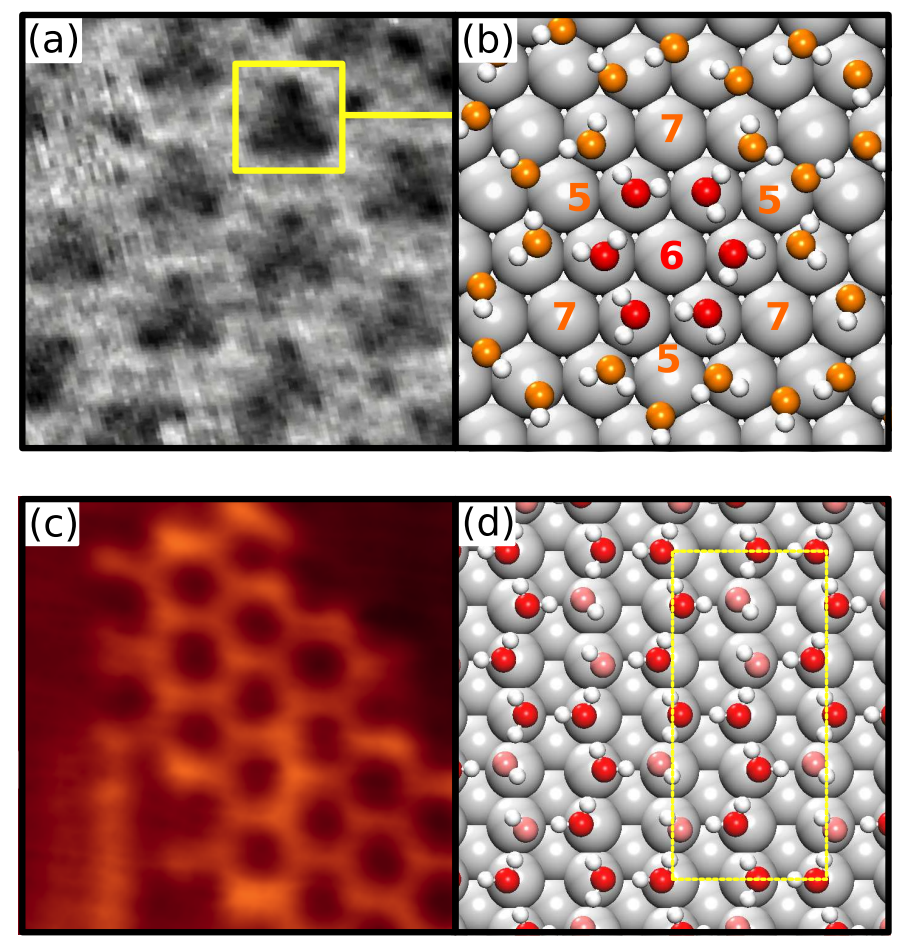

FIG. 5: STM and DFT results for extended water overlayers on two different metal surfaces. STM image (a) of a water adlayer on $\mathrm{Pt}(111)$ and the corresponding DFT-based structural model (b). The structural model is comprised of 5, 6, and 7-membered rings with the water molecules at various heights above the surface (from ref. $\stackrel{23}{ }$ ). STM image (c) of a water-hydroxyl overlayer on $\mathrm{Cu}(110)$ and a DFT-based structural model (d) for this overlayer (from ref. $\underline{50}$ ). The periodic overlayer model is comprised of a $2: 1 \mathrm{H}_{2} \mathrm{O}-\mathrm{OH}$ ratio with a high concentration of Bjerrum $\mathrm{D}$ defects (situations where $2 \mathrm{Hs}$ sit between adjacent $\mathrm{O}$ atoms).

Although ice-like bilayer models have been used to attempt to explain this structure in the past ${ }^{51}$, recent insights reveal a more complex scenario ${ }^{50}$. Interestingly, the overlayer is not composed of a fully $\mathrm{H}$ bonded network, instead it contains Bjerrum defects, where two $\mathrm{H}$ atoms sit between adjacent $\mathrm{O}$ atoms. This arrangement implies that maximizing the number of $\mathrm{H}$ bonds within a wetting overlayer is not a reliable criterion to identify stable structures. In this particular case, the overlayer is stabilized by the formation of strong $\mathrm{H}$ bonds as water donates to hydroxyl, with pairs of non-donor hydroxyls accommodated as defects within the $\mathrm{H}$ bond network (Fig. [5(d)). This explains the formation of a non-stoichiometric overlayer preferentially containing $\left(\mathrm{H}_{2} \mathrm{O}\right)_{2} \mathrm{OH}$ trimers and giving rise to the $2: 1 \mathrm{H}_{2} \mathrm{O}-\mathrm{OH}$ 
ratio. Hydroxyl groups are strong $\mathrm{H}$ bond acceptors, but rather weak donors, a principle that also helps to explain the 1:1 $\mathrm{H}_{2} \mathrm{O}-\mathrm{OH}$ chains that form in the absence of excess water $\frac{52}{}$, as well as the structure of hydroxyl/water phases on other surfaces ${ }^{53}$.

\section{Three-dimensional structures}

On non-wetting surfaces such as $\mathrm{Cu}(111)$ water forms 3D clusters, which show a variety of complex structures, but never a simple $\operatorname{Ih}(0001)$ or Ic(111) bilayer termination $\underline{24}$. Depending on coverage, the structures formed include monomer decorated double bilayers with different super-structures, a faceted surface, pyramidal islands, and nanocrystallites with well defined height. Instead of the simple bilayer termination, a $(2 \times 1)$ superstructure and pyramidal facets of $\operatorname{Ih}(1101)$ or Ic(221) are the most commonly observed terminating motifs. A superstructure was also seen in He atom scattering from ice layers grown on $\operatorname{Pt}(111)^{\frac{54}{4}}$ and has been explained by the ordering of dangling $\mathrm{OH}$ groups in the ice surface to minimise the surface energy $\underline{55}-\underline{57}$.

Metal surfaces that do wet show a range of multilayer adsorption behaviour, depending on how tightly the first layer of water is bound. On several surfaces where stable wetting layers form, such as $\mathrm{Ru}(0001)$ and $\operatorname{Pd}(111)$, adsorption of further water causes 3D ice clusters to grow, rather than a continuous water film $\underline{58,59}$. These surfaces are characterised by having tightly bound first layer water, with no dangling $\mathrm{OH}$ groups to bond to further water. Evidently it is not favourable to disrupt the first layer bonding in order to stabilize multilayer adsorption, so the surface does not form an epitaxial multilayer. On $\operatorname{Pt}(111)$ the situation is different ${ }^{25}, 60$. In this case adsorption of more water produces $3 \mathrm{D}$ islands of hexagonal or cubic ice (Fig. 2(h)), reconstructing the original water film $\underline{61} \underline{62}$. Reconstruction of the first layer is driven by the increased $\mathrm{H}$ bonding to the multilayer, but no detailed models are available for this buried interface, although a similar mechanism also operates on $\mathrm{Ni}(111)^{63}$. Removing uncoordinated $\mathrm{OH}$ groups in the first layer by locking them up in a tightly $\mathrm{H}$ bonded $\mathrm{H}_{2} \mathrm{O}-\mathrm{OH}$ structure prevents the first layer relaxing and instead clusters are formed $\underline{64}$. 


\section{DISCUSSION AND OUTLOOK: QUESTIONS WE CAN AND CANNOT AN- SWER}

The last few years has seen tremendous progress in basic understanding of water at surfaces, as the examples discussed illustrate. Although well-defined surface science style studies can be painstaking they are nonetheless incredibly useful as they reveal precisely at the molecular level how water behaves at interfaces. This is highly valuable information and some of the key questions these studies now allow us to answer, which just a few years ago we could only speculate upon, are:

(i) Where do water molecules preferentially adsorb on metal surfaces? As we have seen, STM reveals that isolated water monomers adsorb above individual metal atoms of the substrate. This is consistent with DFT studies on many close-packed metal surfaces. DFT further predicts that in the most stable orientation the dipole moment of the water molecule is almost parallel to the surface (Fig. 3(c)), so as to facilitate interaction of the lone pair $1 b_{1}$ orbital of water with the substrate. This basic adsorption structure for the water monomer is a key structural element of all water-metal overlayer adsorption models characterised to date. However, perfect 2D H bonded networks cannot be built exclusively from flat-lying water monomers because this would lead to too many $\mathrm{O}-\mathrm{H}$ bonds being parallel to the surface. Thus all extended overlayer structures characterised to date have a combination of flat-lying water monomers at atop sites with other water molecules with their dipoles aligned more or less with the surface normal.

(ii) How strong are interfacial $H$ bonds? The strength and length of $\mathrm{H}$ bonds within metal-adsorbed clusters and overlayers can vary greatly, being either stronger or weaker than $\mathrm{H}$ bonds between water molecules in the gas phase. On the one hand, cooperative forces can lead to stronger $\mathrm{H}$ bonds at the interface with metals. For example, the $\mathrm{H}$ bond length in the water dimer is generally shorter when it is adsorbed on a metal surface (Fig. [3(f)) than it is in the gas phase. This is because the dipole moment of the water molecule donating the $\mathrm{H}$ bond is enhanced through polarisation by the substrate. However, on the other hand, interaction with the surface can weaken the $\mathrm{H}$ bonding within the overlayer as a result of "orbital competition" 1.65 . The alternating O-O bond lengths within the adsorbed water hexamer provide an example of this effect: water molecules that interact strongly with the substrate make relatively poor $\mathrm{H}$ bond acceptors because the water lone pair orbital $\left(1 \mathrm{~b}_{1}\right)$ 
is involved both in bonding with the surface and in accepting $\mathrm{H}$ bonds.

(iii) How important is the number of $H$ bonds to the stability of an overlayer? When it comes to water on metals it's quality and not quantity of $\mathrm{H}$ bonds that matters: the most stable structure is not necessarily the one with the most $\mathrm{H}$ bonds. This is seen clearly in the STM experiments of water hexamers. The experimentally observed structure is the cyclic water hexamer (Fig. 3(g)), yet this hexamer has only $6 \mathrm{H}$ bonds, 2 fewer than the so called cage isomer, which is the most stable isomer in the gas phase. Another example is the $\mathrm{OH}-\mathrm{H}_{2} \mathrm{O}$ overlayer on $\mathrm{Cu}(110)$ that we have just discussed. In this system the $\mathrm{H}$ bond each $\mathrm{OH}$ molecule could in principle donate to an adjacent water molecule has been sacrificed so as to facilitate the reorientation needed to strengthen the $\mathrm{H}$ bonds the $\mathrm{OH}$ accepts from neighbouring water molecules. The implication of this observation is that the adsorbed $\mathrm{OH}$ molecule is a much better acceptor of $\mathrm{H}$ bonds than it is a donor, a conclusion that likely applies to adsorbed $\mathrm{OH}$ groups in general ${ }^{15,66}$.

(iv) Does a bilayer form when water adsorbs on metals? For the best part of the 1980s and 1990s the bilayer model was essentially the "standard model" for water adsorption on metals. However, from the discussion so far it should be clear that when wetting layer structures are examined in detail there is scant evidence for the formation of adsorbed bilayers. The $\sqrt{3}$ diffraction pattern observed on many metals turn out not to be the result of bilayers and indeed are often associated with partially dissociated $\mathrm{OH}-\mathrm{H}_{2} \mathrm{O}$ overlayers instead $^{5}$. The one system for which there is the most compelling evidence so far is water on an alloy of platinum and tin 67 ; a surface with $\sqrt{3}$ corrugation that was specifically tailored to facilitate bilayer adsorption. Despite the widespread assumption that water adsorbs in a bilayer structure, the fact that it doesn't and that there is no general model for overlayer adsorption should not really come as a surprise given the immensely rich phase diagram of water.

Beyond the questions we currently have credible answers to there are many more which we can't yet answer with confidence. Some particular questions that investigators have been thinking about for many years but are now increasingly turning their attention to are:

(i) What are the main factors governing the structure of water overlayers at metal surfaces? Obviously this is $\mathrm{H}$ bonding and water-metal bonding, and for specific systems we can understand how this balance plays out as discussed. However, it would be premature to conclude that we fully understand this balance without, for example, the capacity to 
correctly predict what the wetting layer structure would be for water on some arbitrary unexamined surface. What structures, for example, would 2/3 ML of water form on Rh(110) or 0.5 ML of water on $\operatorname{Pd}(100)$ ? Much more well-defined work combining experiment and theory is needed before the underlying physical principles controlling the structure of water at metals are understood well enough to make confident intuitive predictions about wetting layer structures.

(ii) What happens beyond the first layer? The discussion thus far has largely focussed on the first contact layer between water and metals (at cryogenic temperatures), where there remains much to learn. However, increasingly there is interest in understanding multilayer water adsorption and ideally the structure and dynamics of liquid water films at ambient temperatures. Traditionally the study of water multilayers and liquid water structure has been challenging for both experiment and theory alike. This is still the case, however, recent developments are encouraging. For example, the emergence of high pressure x-ray photoelectron spectroscopy (e.g. Ref. $\frac{68}{}$ ), improvements in atomic force microscopy experiments and analysis for solid-liquid interfaces (e.g. Refs. ${ }^{69}-71$ ), and faster computers and computer codes all bring atomistic understanding of solid-liquid interfaces a step closer $\underline{72} \underline{75}$. Although much work remains to be done, one of the key concepts that has emerged already is that nucleation of a second layer of water on top of the contact layer is facilitated by the presence of $\mathrm{OH}$ groups of water molecules that either stick out of the contact layer away from the surface (so called dangling $\mathrm{OH}$ bonds) or can easily reconstruct to do so in the presence of more water. It will be interesting to see what other concepts emerge over the coming years.

(iii) How can we do a better job with theory? It should be clear by now that in this area there is an almost symbiotic relationship between experiment and theory, with e.g. STM often relying on DFT to come up with plausible low energy structural models. It's only appropriate then to comment on some of the challenges remaining for theory. Generally with computer simulations one wants to tackle larger systems, over longer timescales, and with increased accuracy. Advances in algorithmic efficiency and computational power are consistently making simulations with larger system sizes and over long timescales possible, as illustrated by the recent DFT-based molecular dynamics simulations of solid-liquid interfaces $^{72} \underset{-78}{ }$. Increased efficiency and computational power also makes it much easier to explore configurational space when attempting to characterise surface structures. Indeed "blind" or "random" structure searching, which has proved to be a very valuable approach 
in materials and pharmaceutical science (see e.g. Ref. ${ }^{79}$ ), has great potential to speed up the characterisation of novel surface structures in general and water-ice structures in particular. In terms of increased accuracy, DFT has been remarkably successful in working out the structures of water on metals and more often than not the most stable adsorption structure identified with DFT has matched experiment. However, the standard exchange-correlation functionals used in most routine water adsorption studies (usually generalised-gradient approximation functionals such as PBE and PW91) have various well-known limitations. One of the key limitations is that van der Waals dispersion forces are not accounted for and the lack of dispersion has been a topic of much discussion in this area over the years (see e.g. Ref. $\left.{ }^{80}\right)$. It is encouraging that dispersion-based DFT approaches are now beginning to be applied to water-metal adsorption studies $\underline{80} \underline{\underline{83}}$ and also that electronic structure approaches beyond DFT are emerging for water adsorption, such as the Random Phase Approximation (RPA) and quantum Monte Carlo (QMC) ${ }^{84}$. Another important issue is the role of quantum nuclear effects (zero point motion, quantum tunnelling, and delocalisation). At present the vast majority of theoretical studies treat the nuclei as classical-point-like particles and only electrons are treated quantum mechanically. However, there is growing evidence from experiment and theory that the quantum nature of the proton — particularly in $\mathrm{H}$ bonded configurations such as for water overlayers at surfaces - can have a substantial effect on the structure and dynamics of $\mathrm{H}$ bonds (see e.g. $\underline{2}, 27,85,86$ ).

To conclude, the last few years has seen tremendous progress in fundamental understanding of water at interfaces, with well-defined studies at metal surfaces providing key insight at the molecular level. Important concepts in relation to the structure of water at surfaces have emerged but there remains much to be understood in terms of further developing the basic physical principles that control the structure of water at interfaces and in using these principles to improve technological processes such as ice nucleation inhibition or water desalination. Being able to routinely predict the structure of a water adlayer on a given (metal) surface is certainly something we should soon be able to do and thus we should then be in a position to use this information to identify (or develop) substrates which could be used to e.g. inhibit or accelerate ice nucleation or facilitate very rapid water diffusion for the purposes of desalination. 


\section{ACKNOWLEDGMENTS}

J. C. is a Ramón y Cajal fellow and Newton Alumnus supported by the Spanish Government and The Royal Society, respectively. A. M. is supported by the European Research Council.

1 Michaelides, A. \& Morgenstern, K. Ice nanoclusters at hydrophobic metal surfaces. Nature Mater. 6, 597 (2007).

2 Kumagai, T., Okuyama, H., Hatta, S., Aruga, T. \& Hamada, I. H-atom relay reactions in real space. Nature Mater. 11, 167 (2012).

3 Yates Jr., J. T. \& Campbell, C. T. Surface chemistry: Key to control and advance myriad technologies. Proc. Natl. Acad. Sci. USA 108, 911 (2011).

4 Henderson, M. A. The interaction of water with solid surfaces: fundamental aspects revisited. Surf. Sci. Rep. 46, 5 (2002).

5 Hodgson, A. \& Haq, S. Water adsorption and the wetting of metal surfaces. Surf. Sci. Rep. 64, 381 (2009).

6 Feibelman, P. J. Concluding remarks. Faraday Discuss. 141, 467 (2009).

7 Feibelman, P. J. The first wetting layer on a solid. Phys. Today 63, 34 (2010).

8 Michaelides, A. Density functional theory simulations of water-metal interfaces: waltzing waters, a novel 2D ice phase, and more. Appl. Phys. A 85, 415 (2006).

9 Doering, D. L. \& Madey, T. E. The adsorption of water on clean and oxygen-dosed $\mathrm{Ru}(001)$. Surf. Sci. 123, 305 (1982).

10 Ogasawara, H. et al. Structure and bonding of water on Pt(111). Phys. Rev. Lett. 89, 276102 (2002).

11 Thiel, P. A. \& Madey, T. E. The interaction of water with solid-surfaces - Fundamental-aspects. Surf. Sci. Rep. 7, 211 (1987).

12 Held, G. \& Menzel, D. The structure of the $p(\sqrt{3} \times \sqrt{3}) R 30^{\circ}$ bilayer of $\mathrm{D}_{2} \mathrm{O}$ on $\mathrm{Ru}(001)$. Surf. Sci. 316, 92 (1994).

13 Held, G. et al. Light-atom location in adsorbed benzene by experiment and theory. Phys. Rev. Lett. 87, 216102 (2001). 
14 Feibelman, P. J. Partial dissociation of water on Ru(0001). Science 295, 99 (2002).

15 Michaelides, A. \& Hu, P. A density functional theory study of hydroxyl and the intermediate in the water formation reaction on Pt. J. Chem. Phys. 114, 513 (2001).

16 Michaelides, A. \& Hu, P. Catalytic water formation on platinum: A first-principles study. $J$. Am. Chem. Soc. 123, 4235 (2001).

17 Gallagher, M., Omer, A., Darling, G. \& Hodgson, A. Order and disorder in the wetting layer on $\mathrm{Ru}(0001)$. Faraday Disc. Chem. Soc. 141, 231 (2009).

18 Gawronski, H., Carrasco, J., Michaelides, A. \& Morgenstern, K. Manipulation and control of hydrogen bond dynamics in absorbed ice nanoclusters. Phys. Rev. Lett. 101, 136102 (2008).

19 Tatarkhanov, M. et al. The structure of mixed $\mathrm{H}_{2} \mathrm{O}-\mathrm{OH}$ monolayer films on $\mathrm{Ru}(0001)$. J. Chem. Phys. 129, 154109 (2008).

20 Yamada, T., Tamamori, S., Okuyama, H. \& Aruga, T. Anisotropic water chain growth on $\mathrm{Cu}(110)$ observed with scanning tunneling microscopy. Phys. Rev. Lett. 96, 036105 (2006).

21 Cerda, J. et al. Novel water overlayer growth on $\operatorname{Pd}(111)$ characterized with scanning tunneling microscopy and density functional theory. Phys. Rev. Lett. 93, 116101 (2004).

22 Salmeron, M. et al. Water growth on metals and oxides: binding, dissociation and role of hydroxyl groups. Faraday Discuss. 141, 221 (2009).

23 Nie, S., Feibelman, P. J., Bartelt, N. C. \& Thürmer, K. Pentagons and Heptagons in the First Water Layer on Pt(111). Phys. Rev. Lett. 105, 026102 (2010).

24 Mehlhorn, M. \& Morgenstern, K. Faceting during the Transformation of Amorphous to Crystalline Ice. Phys. Rev. Lett. 99, 246101 (2007).

25 Thürmer, K. \& Bartelt, N. C. Nucleation-limited dewetting of ice films on Pt(111). Phys. Rev. Lett. 100, 186101 (2008).

26 Motobayashi, K., Matsumoto, C., Kim, Y. \& Kawai, M. Vibrational study of water dimers on Pt(111) using a scanning tunneling microscope. Surf. Sci. 602, 3136 (2008).

27 Kumagai, T. et al. Tunneling dynamics of a hydroxyl group adsorbed on $\mathrm{Cu}(110)$. Phys. Rev . $B$ 79, 035423 (2009).

28 Michaelides, A., Ranea, V. A., de Andres, P. L. \& King, D. A. General model for water monomer adsorption on close-packed transition and noble metal surfaces. Phys. Rev. Lett. 90, 216102 (2003).

29 Ranea, V. A. et al. Water dimer diffusion on $\operatorname{Pd}(111)$ assisted by an H-bond donor-acceptor 
tunneling exchange. Phys. Rev. Lett. 92, 136104 (2004).

30 Meng, S., Wang, E. G. \& Gao, S. W. Water adsorption on metal surfaces: A general picture from density functional theory studies. Phys. Rev. B 69, 195404 (2004).

31 Carrasco, J., Michaelides, A. \& Scheffler, M. Insight from first principles into the nature of the bonding between water molecules and 4d metal surfaces. J. Chem. Phys. 130, 184707 (2009).

32 Okuyama, H. \& Hamada, I. Hydrogen-bond imaging and engineering with a scanning tunnelling microscope. J. Phys. D: Appl. Phys. 44, 464004 (2011).

33 Kumagai, T. et al. Direct observation of hydrogen-bond exchange within a single water dimer. Phys. Rev. Lett. 100, 166101 (2008).

34 Kumagai, T., Okuyama, H., Hatta, S., Aruga, T. \& Hamada, I. Water clusters on Cu(110): Chain versus cyclic structures. J. Chem. Phys. 134, 024703 (2011).

35 Mitsui, T., Rose, M. K., Fomin, E., Ogletree, D. F. \& Salmeron, M. Water diffusion and clustering on $\operatorname{Pd}(111)$. Science 297, 1850 (2002).

36 Morgenstern, K. Scanning tunnelling microscopy investigation of water in submonolayer coverage on $\operatorname{Ag}(111)$. Surf. Sci. 504, 293 (2002).

37 Morgenstern, K. \& Nieminen, J. Intermolecular bond length of ice on $\mathrm{Ag}(111)$. Phys. Rev. Lett. 88, $066102(2002)$.

38 Mehlhorn, M., Carrasco, J., Michaelides, A. \& Morgenstern, K. Local Investigation of Femtosecond Laser Induced Dynamics of Water Nanoclusters on $\mathrm{Cu}(111)$. Phys. Rev. Lett. 103, 026101 (2009).

39 Santra, B. et al. On the accuracy of density-functional theory exchange-correlation functionals for $\mathrm{H}$ bonds in small water clusters. II. The water hexamer and van der Waals interactions. $J$. Chem. Phys. 129, 194111 (2008).

40 Haq, S., Clay, C., Darling, G. R., Zimbitas, G. \& Hodgson, A. Growth of intact water ice on $\mathrm{Ru}(0001)$ between 140 and $160 \mathrm{~K}$ : Experiment and density-functional theory calculations. Phys. Rev. B 73, 115414 (2006).

41 Lee, J., Sorescu, D. C., Jordan, K. D. \& Jr., J. T. Y. Hydroxyl chain formation on the Cu(110) surface: watching water dissociation. J. Phys. Chem. C 112, 17672 (2008).

42 Carrasco, J. et al. A one-dimensional ice structure built from pentagons. Nature Mater. 8, 427 (2009).

43 Ma, B.-Q., Sun, H.-L. \& Gao, S. Cyclic water pentamer in a tape-like structure. Chem. 
Commun. 2220 (2004).

44 Naskar, J. P., Drew, M. G. B., Hulme, A., Tocher, D. A. \& Datta, D. Occurrence of ribbons of cyclic water pentamers in a metallo-organic framework formed by spontaneous fixation of $\mathrm{CO}_{2}$. Cryst. Eng. Comm. 7, 67 (2005).

45 Verdaguer, A., Sacha, G. M., Bluhm, H. \& Salmeron, M. Molecular structure of water at interfaces: Wetting at the nanometer scale. Chem. Rev. 106, 1478 (2006).

46 McBride, F. et al. Strain Relief and Disorder in Commensurate Water Layers Formed on Pd(111). J. Phys.: Condens. Matter 24, 124102 (2012).

47 Glebov, A., Graham, A. P., Menzel, A. \& Toennies, J. P. Orientational ordering of twodimensional ice on Pt(111). J. Chem. Phys. 106, 9382 (1997).

48 Standop, S., Redinger, A., Morgenstern, M., Michely, T. \& Busse, C. Molecular structure of the $\mathrm{H}_{2} \mathrm{O}$ wetting layer on $\mathrm{Pt}(111)$. Phys. Rev. B 82, 161412 (2010).

49 Feibelman, P. J., Bartelt, N. C., Nie, S. \& Thürmer, K. Interpretation of high-resolution images of the best-bound wetting layers on Pt(111). J. Chem. Phys. 133, 154703 (2010).

50 Forster, M., Raval, R., Hodgson, A., Carrasco, J. \& Michaelides, A. c(2 x 2) Water-Hydroxyl Layer on $\mathrm{Cu}(110)$ : A Wetting Layer Stabilized by Bjerrum Defects. Phys. Rev. Lett. 106, 046103 (2011).

51 Ren, J. \& Meng, S. Atomic structure and bonding of water overlayer on $\mathrm{Cu}(110)$ : The borderline for intact and dissociative adsorption. J. Am. Chem. Soc. 128, 9282 (2006).

52 Forster, M., Raval, R., Carrasco, J., Michaelides, A. \& Hodgson, A. Water-hydroxyl phases on an open metal surface: breaking the ice rules. Chem. Sci. 3, 93 (2012).

53 Schiros, T. et al. Cooperativity in Surface Bonding and Hydrogen Bonding of Water and Hydroxyl at Metal Surfaces. J. Phys. Chem. C 114, 10240 (2010).

54 Glebov, A., Graham, A. P., Menzel, A. \& Toennies, J. P. A helium atom scattering study of the structure and phonon dynamics of the ice surface. J. Chem. Phys. 112, 11011 (2000).

55 Buch, V., Groenzin, H., Lit, I., Shultz, M. \& Tosatti, E. Proton order in the ice crystal surface. Proc. Natl. Acad. Sci. USA 105, 5969 (2008).

56 Pan, D. et al. Surface energy and surface proton order of ice Ih. Phys. Rev. Lett. 101, 155703 (2008).

57 Pan, D. et al. Surface energy and surface proton order of the ice Ih basal and prism surfaces. J. Phys. Cond. Mat. 22, 074209 (2010). 
58 Haq, S. \& Hodgson, A. Multilayer growth and wetting of $\mathrm{Ru}(0001)$. J. Phys. Chem. C 111, $5946(2007)$.

59 Kimmel, G., Petrik, N., Dohnalek, Z. \& Kay, B. Crystalline ice growth on Pt(111) and Pd(111): Nonwetting growth on a hydrophobic water monolayer. J. Chem. Phys. 126, 114702 (2007).

60 Thürmer, K. \& Bartelt, N. C. Growth of multilayer ice films and the formation of cubic ice imaged with STM. Phys. Rev. B 77, 195425 (2008).

61 Zimbitas, G., Haq, S. \& Hodgson, A. The structure and crystallization of thin water films on Pt(111). J. Chem. Phys. 123, 174701 (2005).

62 Zimbitas, G. \& Hodgson, A. The morphology of thin water films on Pt(111) probed by chloroform adsorption. Chem. Phys. Lett. 417, 1 (2006).

63 Gallagher, M., Omer, A., Haq, S. \& Hodgson, A. Water monolayer and multilayer adsorption on $\mathrm{Ni}(111)$. Surf. Sci. 601, 268 (2007).

64 Zimbitas, G., Gallagher, M. E., Darling, G. R. \& Hodgson, A. Wetting of mixed OH/ $\mathrm{H}_{2} \mathrm{O}$ layers on $\operatorname{Pt}(111)$. J. Chem. Phys. 128, 074701 (2008).

65 Michaelides, A. Simulating ice nucleation, one molecule at a time, with the DFT microscope. Faraday Disc. 136, 287 (2007).

66 Karlberg, G. S., Olsson, F. E., Persson, M. \& Wahnstrom, G. Energetics, vibrational spectrum, and scanning tunneling microscopy images for the intermediate in water production reaction on $\operatorname{Pt}(111)$ from density functional calculations. J. Chem. Phys. 119, 4865 (2003).

67 McBride, F., Darling, G. R., Pussi, K. \& Hodgson, A. Tailoring the Structure of Water at a Metal Surface: A Structural Analysis of the Water Bilayer Formed on an Alloy Template. Phys. Rev. Lett. 106, 226101 (2011).

68 Yamamoto, S. et al. In situ x-ray photoelectron spectroscopy studies of water on metals and oxides at ambient conditions. J. Phys.: Condens. Matter 20, 184025 (2008).

69 Voitchovsky, K., Kuna, J. J., Contera, S. A., Tosatti, E. \& Stellacci, F. Direct mapping of the solid-liquid adhesion energy with subnanometre resolution. Nature Nanotech. 5, 401 (2010).

70 Fukuma, T. Water distribution at solid-liquid interfaces visualized by frequency modulation atomic force microscopy. Sci. Technol. Adv. Mater. 11, 033003 (2010).

71 Watkins, M., Berkowitz, M. L. \& Shluger, A. L. Role of water in atomic resolution AFM in solutions. Phys. Chem. Chem. Phys. 13, 12584 (2011).

72 Liu, L., Krack, M. \& Michaelides, A. Density oscillations in a nanoscale water film on salt: 
Insight from ab initio molecular dynamics. J. Am. Chem. Soc. 130, 8572 (2008).

73 Schnur, S. \& Gross, A. Properties of metal-water interfaces studied from first principles. New J. Phys. 11, 125003 (2009).

74 Ikeshoji, T., Otani, M., Hamada, I. \& Okamoto, Y. Reversible redox reaction and water configuration on a positively charged platinum surface: first principles molecular dynamics simulation. Phys. Chem. Chem. Phys. 13, 20223 (2011).

75 Liu, L., Laio, A. \& Michaelides, A. Initial stages of salt crystal dissolution determined with ab initio molecular dynamics. Phys. Chem. Chem. Phys. 13, 13162 (2011).

76 Liu, L., Changjun, Z., Thornton, G. \& Michaelides, A. Structure and dynamics of liquid water on rutile $\mathrm{TiO}_{2}(110)$. Phys. Rev. B 82, 161415 (2008).

77 Sharma, M., Donadio, D., Schwegler, E. \& Galli, G. Probing Properties of Water under Confinement: Infrared Spectra. Nano Lett. 8, 2959 (2008).

78 Cicero, G., Grossman, J. C., Schwegler, E., Gygi, F. \& Galli, G. Water Confined in Nanotubes and between Graphene Sheets: A First Principle Study. J. Am. Chem. Soc. 130, 1871 (2008).

79 Pickard, C. \& Needs, R. J. Ab initio random structure searching. J. Phys.: Condens. Matter. 23, 053201 (2011).

80 Carrasco, J., Santra, B., Klimeš, J. \& Michaelides, A. To Wet or Not to Wet? Dispersion Forces Tip the Balance for Water Ice on Metals. Phys. Rev. Lett. 106, 026101 (2011).

81 Hamada, I., Lee, K. \& Morikawa, Y. Interaction of water with a metal surface: Importance of van der Waals forces. Phys. Rev. B 81, 115452 (2010).

82 Lew, W., Crowe, M. C., Campbell, C. T., Carrasco, J. \& Michaelides, A. The Energy of Hydroxyl Coadsorbed with Water on Pt(111). J. Phys. Chem. C 115, 23008 (2011).

83 Tonigold, K. \& Gross, A. Dispersive interactions in water bilayers at metallic surfaces: A comparison of the PBE and RPBE functional including semiempirical dispersion. J. Comp. Chem. 33, 695 (2012).

84 Ma, J. et al. Adsorption and diffusion of water on graphene from first principles. Phys. Rev. B 84, $033402(2011)$.

85 Li, X.-Z., Probert, M. I. J., Alavi, A. \& Michaelides, A. Quantum Nature of the Proton in Water-Hydroxyl Overlayers on Metal Surfaces. Phys. Rev. Lett. 104, 066102 (2010).

86 Li, X.-Z., Walker, B. \& Michaelides, A. Quantum nature of the hydrogen bond. Proc. Natl. Acad. Sci. USA 108, 6369 (2011). 